\title{
Keeping Quiet: Factors that Hinder Anger Expression at Work
}

\author{
Lisa L. Stickney \\ University of Baltimore, U.S.A. \\ 1stickney@ubalt.edu \\ Deanna Geddes \\ Temple University, U.S.A. \\ geddes@temple.edu
}

\author{
Paper Presented at the \\ $24^{\text {th }}$ Annual International Association of Conflict Management Conference \\ Istanbul, Turkey \\ July 3-6, 2011
}

\begin{abstract}
More research is emerging that finds workplace anger expression (versus suppression) is more likely to promote positive individual and organizational outcomes. However, many angry employees at work choose to remain silent rather than express their anger. What factors contribute most to this decision? Using content and factor analysis, we identify three key categories of factors that hinder anger expression at work, most to least frequent, respectively: professional courtesy, fear of repercussions, and organizational norms. We argue that rather than self-focused concerns of retaliation or sanctioning, most people fail to speak up when angry because of perceptions that "professionals" do not express anger, or the concern that anger expression shows a lack of respect for fellow organizational members. Although this is admirable in its other-oriented (versus self-centered) focus, there is a false assumption that keeping one's anger and concerns hidden about a problematic workplace situation is helpful for the organization. Management can play a key role in changing social norms to allow for honest and helpful expressions of anger by employees without fear of appearing unprofessional or receiving organizational sanctions.
\end{abstract}


We know that organizational members get angry at work. Perceived injustices, inefficiencies, norm violations, blocked goals and even another's anger expression can trigger intense feelings of anger (Clore \& Centerbar, 2004; VanderVoort \& Ragland, 1996). Research studies show that workplace anger expression (versus suppression) is more likely to promote positive individual and organizational outcomes (Geddes \& Stickney, 2011; Gibson \& Callister, 2010). Even when anger intensity, duration, or form constitutes what organizational observers consider going "over the line" of propriety, expressed anger signals to organizational members an opportunity to respond effectively toward a triggering event, the angry employee, or both (Geddes \& Callister, 2007).

Less understood is why people choose not to express felt anger at work. In other words, why do people remain silent, rather than express anger? There are likely several factors that hinder the expression of anger at work ranging from individual traits (introversion, fears) to environmental factors (organizational emotion display norms, sanctions) (Böddeker \& Stemmler, 2000; Gross \& Levenson, 1993; Rafaeli \& Sutton, 1989). Intensity is perhaps the most studied variable characterizing felt and expressed anger, and a contributing factor for anger expression. That is, research shows that with more intense anger, individuals are more motivated (or less inhibited) to express their anger (Frijda, Ortony, Sonnemans, \& Clore, 1992). This implies that with lower intensity felt anger, employees should be less likely to express anger. However, even with highly intense felt emotion, individuals often choose not to express their anger (Ashkanasy, Zerbe, \& Hartel, 2002; Richards \& Gross, 1999; Tavris, 1984). So what prevents people, especially those who are not "emotional laborers" (Hochschild, 1983; Scott \& Myers, 2005) from expressing anger at work? 
In a recent pilot study, we asked this question, initially through an open ended questionnaire. The survey went to 32 full-time, office workers of a large public utility subsidiary. Thirteen employees completed and returned usable surveys. Participants provided both specific and general attitudes toward anger and its expression in the organization. Our key open-ended question was "What hinders or prevents you from expressing anger at work?" Both authors transcribed and examined participant responses. The unit of analysis was defined as a distinguishable action. Once the qualitative data were unitized with 100\% agreement (see Guetzkow, 1950), both researchers operating separately content analyzed responses to the respective question, identifying factors based on description similarity. After comparing and labeling our respective categories of anger hindering factors, the few differences were discussed until there was 100\% agreement. Our analysis identified 7 factors that can hinder anger expression at work: fear of co-worker repercussions, fear of management repercussions, professionalism, impression management, respect for others, organizational climate/environment, and "nothing prevents me from expressing anger at work."

These became categories for a larger survey (Study 1). With this second data collection, we could assess the adequacy of our anger-hindering items, expand them if possible, and generate some preliminary results. We revised our survey to include the categories identified from the pilot study and we also included a write-in "Other" option for this question as well. We distributed this survey to 196 participants from a small aerospace and a mechanical services company, advanced (junior or senior) undergraduate business majors, and employed MBA students. All participants worked at least part-time, and 56 percent worked full-time. Fifty-nine percent were female and most (73\%) were Caucasian and under the age of $30(70 \%)$. Thirty-one percent had held their current job for less than a year, 36 percent for more than one year but less 
than three years, 16 percent between three and five years, and 17 percent were employed over five years at their current position. They completed the surveys at work or in class, returned them promptly to the researchers, and were assured anonymity and confidentiality.

Based on the results of the data set, we expanded our response options, an additional item: management responsible for anger. This modified response set was included in the finalized "Emotions in the Workplace" questionnaire used for Study 2. Participants in this study included 194 individuals who acknowledged experiencing anger at work. All were volunteers and full-time U.S. employees recruited from an eastern healthcare center (26\%), graduate business classes in a large eastern U.S. university (52\%), and working associates of students attending two eastern U.S. business schools (22\%). Fifty-nine percent were female and 87 percent were Caucasian.

Frequencies were compiled for each option on both surveys. Items selected by respondents from both Study 1 and Study 2 also were factor analyzed to identify primary categories of factors that hinder anger expression (see Tables $1,2 \& 3$ ).

Results of this preliminary analysis suggest that there are three key categories of factors that hinder anger expression: fear of repercussions, professional courtesy, and salient cultural norms. Most significant in our findings was the fact that people are more likely to suppress anger on the presumption of professionalism and respect for others, rather than a fear of sanctions or negative repercussions. Nevertheless, when they do fear negative repercussions, they are most concerned about how management will react. Further, cultural norms, reflected in the organizational climate/environment and experience with a lack of response by management, also affect whether or not one's inclination to express anger is "hindered." 
Implications of our findings suggest that a significant number of employees fail to express their anger, not because of self-focused concerns, but because of perceptions that "professionals" do not express anger, or the concern that anger expression shows a lack of respect to fellow organizational members. Although this is admirable in its other-oriented focus, there is a false assumption that keeping one's anger and concerns hidden about a problematic workplace situation is helpful for the organization. In fact, if the infuriating episode is not addressed — including appropriate individuals identified and notified of the problem—it will likely continue. This may in turn create additional problems for the angry employee, their colleagues, and the organization itself, for as long as it remains unaddressed. The "professional courtesy" hindering factor also suggests that previous norms and/or training may be sending the wrong message with regard to anger expression. Often anger expression is identified as deviance or aggression. Nevertheless, if organizational members - both agents and observers- recognize potential positive and prosocial aspects of anger, they may be less inhibited and more willing to speak up when angry, and less likely to sanction those that do. Changing perceptions of what constitutes professionalism and courtesy can change organizational norms so that people are more willing to speak up, rather to remain silent, when the workplace needs change.

Research suggests that managers and co-workers who provide more supportive responses to anger expression promote favorable situational change at work, while sanctioning anger or doing nothing following expressed anger, does not (Geddes \& Stickney, 2011). This study also found that supportive work environments were significantly related to supportive responses by management. Thus, one way to reduce repercussion fears that hinder anger expression is to train managers to understand that anger displays are often not deviance nor insubordination, but honest, potentially helpful signals identifying problematic conditions that require their attention. 
Organizational cultures and climates are highly influenced by management action toward employees (Schein, 2004). Thus, when management responds to angry employees in a more supportive than sanctioning manner, they likely help establish and reinforce cultural norms and work environments that can allow anger and professionalism to coexist, and ultimately, promote more tolerant, concerned responses toward emotional organizational members.

\section{REFERENCES}

Ashkanasy, N. M., Zerbe, W. J., \& Hartel, C. E. J. 2002. Managing emotions in the workplace. In N. M. Ashkanasy \& W. J. Zerbe \& C. E. J. Hartel (Eds.), Managing emotions in the workplace: 3-24. Armonk, NY: M. E. Sharpe, Inc.

Böddeker, I. \& Stemmler, G. 2000. Who responds how and when to anger? The assessment of actual anger response styles and their relation to personality. Cognition and Emotion, 14(6): 737762.

Clore, G. L. \& Centerbar, D. B. 2004. Analyzing Anger: How to Make People Mad. Emotion, 4(2): 139-144.

Frijda, N. H., Ortony, A., Sonnemans, J., \& Clore, G. L. 1992. The complexity of intensity: Issues concerning the structure of emotional intensity. In M. S. Clark (Ed.), Emotion: 60-89. Newbury Park, CA: Sage.

Geddes, D. \& Callister, R. R. 2007. Crossing the line(s): A dual threshold model of expressing anger in organizations. Academy of Management Review, 32(3): 721-746.

Geddes, D. \& Stickney, L. T. 2011. The trouble with sanctions: Organizational responses to deviant anger displays at work. Human Relations, 64(2).

Gibson, D. E. \& Callister, R. R. 2010. Anger in organizations: Review and integration. Journal of Management, 36(1): 66-93.

Gross, J. J. \& Levenson, R. W. 1993. Emotional suppression: Physiology, self-report, and expressive behavior. Journal of Personality and Social Psychology, 64(6): 970-986.

Guetzkow, H. 1950. Unitizing and categorizing problems in coding qualitative data. Journal of Clinical Psychology, 6: 47-58.

Hochschild, A. 1983. The managed heart: Commercialization of human feeling. Berkeley, CA: University of California Press. 
Rafaeli, A. \& Sutton, R. I. 1989. The expression of emotion in organizational life. In L. L. Cummings \& B. M. Staw (Eds.), Research in organizational behavior, Vol. 11: 1-42. Greenwich, CT: JAI Press.

Richards, J. M. \& Gross, J. J. 1999. Composure at any cost? The cognitive consequences of emotion suppression. Personality and Social Psychology Bulletin, 25: 1033-1044.

Schein, E. H. 2004. Organizational culture and leadership. The Jossey-Bass Business \& Management Series. 3rd ed. San Francisco: Jossey-Bass.

Scott, C. \& Myers, K. K. 2005. The Socialization of Emotion: Learning Emotion Management at the Fire Station. Journal of Applied Communication Research, 33(1): 67-92.

Tavris, C. 1984. On the wisdom of counting to ten: Personal and social dangers of anger expression. In P. Shaver (Ed.), Review of personality and social psychology, Vol. 5: 170-192. Newbury Park, CA: Sage.

VanderVoort, D. J. \& Ragland, D. R. 1996. Expressed and suppressed anger and health problems among transit workers. Current Psychology, 15(2): 179-193.

\section{TABLES}

Table 1. Frequencies of Items that Hinder Anger Expression at Work and Overall Categories of Anger Hindering Factors

\begin{tabular}{|l|l|l|l|}
\hline Survey Items & Study 1 & Study 2 & Hindering Categories \\
\hline Fear of co-worker repercussions & $14 \%$ & $23 \%$ & Fear of personal \\
repercussions (self focus)
\end{tabular}


Table 2. Study 1 Analysis of Factors Hindering Anger Expression

\begin{tabular}{|c|c|c|c|}
\hline & \multicolumn{3}{|c|}{ Component } \\
\hline & 1 & 2 & 3 \\
\hline Hinders: Coworker repercussions & .636 & & -.319 \\
\hline Hinders: Mgmt repercussions & .720 & & \\
\hline Hinders: Professionalism & & .791 & \\
\hline Hinders: Impression Mgmt & .584 & & \\
\hline Hinders: Org. Climate/Environment & & & .856 \\
\hline Hinders: Respect for Others & & .551 & -.367 \\
\hline Hinders: No Inhibitions & -.377 & -.667 & -.371 \\
\hline
\end{tabular}

Principal Component Analysis with Varimax Rotation

Table 3. Study 2 Analysis of Factors Hindering Anger Expression

\begin{tabular}{|l|r|r|r|}
\hline & \multicolumn{3}{|c|}{ Component } \\
\cline { 2 - 4 } & \multicolumn{1}{|c|}{1} & \multicolumn{1}{|c|}{2} & \multicolumn{1}{c|}{3} \\
\hline Hinders: Coworker repercussions & .699 & & \\
Hinders: Mgmt repercussions & .739 & & \\
Hinders: Professionalism & & .800 & \\
Hinders: Impression Mgmt & .540 & & \\
Hinders: Org. Climate/Environment & & & -.641 \\
Hinders: Respect for co-workers & & .682 & \\
Hinders: Person evoking anger is mgr & .445 & -.320 & -.470 \\
Hinders: No Inhibitions & -.403 & -.510 & .445 \\
\hline
\end{tabular}

Principal Component Analysis with Varimax Rotation 